\title{
Metabolitos secundarios con actividad medicinal extraídos de hongos provenientes de Centroamérica
}

\section{Secondary metabolites with medicinal activity extracted from fungi found in Central America}

Luis Felipe Arce-Torres ${ }^{1}$, Isabella Gómez-Díaz², Mariana Monge-Artavia ${ }^{3}$, Joselyn Prado-Cordero ${ }^{4}$

Arce-Torres, L; Gómez-Díaz, I; Monge-Artavia, M; PradoCordero. J. Metabolitos secundarios con actividad medicinal extraídos de hongos provenientes de Centroamérica. Tecnología en Marcha. Vol. 33-3. Julio-Setiembre 2020. Pág 80-89.

doi) https://doi.org/10.18845/tm.v33i3.4416

Fecha de recepción: 24 de julio de 2019

Fecha de aprobación: 2 de octubre de 2019

1 Colegio Científico Costarricense sede Cartago, Costa Rica. Correo electrónico: felipe14arce@gmail.com (D) https://orcid.org/0000-0003-3090-8530

2 Instituto Tecnológico de Costa Rica. Cartago, Costa Rica. Correo electrónico: isigd97@gmail.com (iD https://orcid.org/0000-0001-8300-0900

3 Instituto Tecnológico de Costa Rica. Cartago, Costa Rica. Correo electrónico: marige97@gmail.com (D) https://orcid.org/0000-0002-2798-7939

4 Instituto Tecnológico de Costa Rica. Cartago, Costa Rica. Correo electrónico: joselynprado97@gmail.com 


\section{Palabras clave}

Penicilina; antibiótico; triterpenos; polisacáridos; anticancerígeno; antioxidante; psilocibina; psicoterapia.

\section{Resumen}

Existe una gran diversidad de hongos que poseen actividad medicinal en Centroamérica y de ellos se pueden extraer los metabolitos secundarios responsables y adecuados para el tratamiento de distintas enfermedades. En esta revisión se realizó una investigación acerca de seis hongos específicos que se utilizan para el tratamiento de padecimientos físicos: Penicillium chrysogenum (P. chrysogenum), Ganoderma lucidum (G. Iucidum), Trametes versicolor (T. versicolor), Schizophyllum commune (S. commune), Pleurotus mutilus (P. mutilus), Lentinula edodes (L. edodes), y un último hongo que tiene propiedades alucinógenas útiles en el tratamiento de enfermedades mentales: Psilocybe cubensis (P. cubensis). Se encontró que los metabolitos secundarios de mayor importancia para la industria médica son la penicilina y la tiamulina como antibióticos, los triterpenos por su actividad anticancerígena, diversos tipos de polisacáridos que tienen actividad antioxidante, antiparasitarios, estimulantes del sistema inmune y reguladores del colesterol. Asimismo, se estudió el alucinógeno psilocibina y su potencial en tratamientos de psicoterapia.

\section{Keywords}

Penicillin; antibiotic; triterpenes; polysaccharides; anticarcinogenic; antioxidant; psilocybin; psychotherapy.

\section{Abstract}

There's a wide diversity of fungi that have medicinal activity in Central America, from which the secondary metabolites responsible for that activity can be extracted in order to treat various diseases. In this review, six different fungi with the potential to treat physical disorders were studied: Penicillium chrysogenum (P. chrysogenum), Ganoderma lucidum (G. lucidum), Trametes versicolor (T. versicolor), Schizophyllum commune (S. commune), Pleurotus mutilus (P. mutilus), Lentinula edodes (L. edodes), and one hallucinogenic mushroom useful in the field of mental health issues: P. cubensis. The secondary metabolites of greatest importance were penicillin and tiamulin as antibiotics, triterpenes with anticarcinogenic activity, various polysaccharides with antioxidant and antiparasitic activity, immune boosting and cholesterol regulating properties. Additionally, the hallucinogen psilocybin and its potential in psychotherapy treatments was also studied.

\section{Introducción}

Esta revisión bibliográfica corresponde al trabajo realizado para el curso electivo "Procesos Biotecnológicos con el Empleo de Hongos" de la carrera de Ing. en Biotecnología de la Escuela de Biología del Instituto Tecnológico de Costa Rica e impartido por el M.Sc. William Rivera.

A lo largo de los años el ser humano ha tratado de identificar a todos los seres vivos que habitan el planeta Tierra para poder diferenciarlos según su utilidad, perjuicio o solamente para conocer su entorno. Debido a esto, el ser humano ha ido evolucionando en conjunto con su entorno, desde la fabricación de las herramientas para la caza hasta la domesticación de especies de 
flora comestibles. Des de tiempos remotos, los humanos han dependido, transformado y adecuado el entorno en donde se desarrollan para su bien, logrando alcanzar grandes beneficios [1].

Un grupo muy importante de organismos que han evolucionado con el pasar de los años junto con el hombre, es el de los hongos. Estos organismos son seres eucariotas pertenecientes al reino Fungi, algunas de sus características son que no poseen clorofila y pueden encontrarse en 4 niveles de organización (células libres, pseudomicelio, micelio y cuerpo fructífero). Además de esto, poseen dos tipos principales de morfología: unicelulares o filamentosos. A los hongos se les atribuyen múltiples aplicaciones entre las cuales destacan el aprovechamiento en la medicina y en la industria alimenticia [2]. Dentro de los más primitivos usos de los hongos se encuentra la producción de pan, cerveza y vino en civilizaciones tan antiguas como la Egipcia; en estas culturas, pese al desconocimiento a priori del porqué de los procesos, se realizaban fermentaciones para potabilizar el agua y poder tomarla [3].

Dentro de la diversidad fúngica, existen tanto hongos patógenos como beneficiosos para los seres humanos, de los beneficios principales de estos organizamos se pueden destacar dos descubrimientos que fueron clave: Agostino Bassi en 1835 descubrió que la muscardina del gusano de seda era ocasionada por un hongo llamado Beauveria bassiana (hongo entomopatógeno) y Alexander Fleming en 1928 que encontró el efecto antibiótico de la penicilina [4].

Por su parte, dentro de los principales efectos perjudiciales de los hongos se puede mencionar que algunos causan padecimientos como reacciones de hipersensibilidad (alergias), micosis e infecciones (micotoxicosis y micetismos), además, existen hongos tóxicos o venenosos que pueden ocasionar severas lesiones y hasta la muerte al ser ingeridos [5]. Por otra parte, estos organismos son los principales productores de compuestos de uso médico como antibióticos, antioxidantes, anticancerígenos y otros. La producción a gran escala de estos compuestos es una parte muy fuerte de las industrias médica, farmacéutica y nutracéutica, en donde se aplican procesos biotecnológicos [6]. En la presente revisión bibliográfica se hablará de algunos hongos encontrados en Centroamérica que producen compuestos con actividad medicinal importante

\section{Penicillium chrysogenum}

Penicillium chrysogenum (P. chrysogenum) es un hongo filamentoso perteneciente al filo Ascomicota y a la familia de los Trichocomaceae. Este microorganismo se encuentra en varios hábitats, donde crece principalmente en sustratos como pan y frutos cítricos en forma de colonias verde azuladas. Dentro de los ecosistemas que coloniza también se mencionan edificaciones con ambientes húmedos y mohosos, en las cuales su esporas germinan y colonizan los ambientes en cuanto se dan las condiciones favorables para desarrollarse, producen el micelio del hongo y este es capaz de deteriorar diferentes materiales produciendo grandes daños a la infraestructura $[7]$.

Aunado a esto, también puede ocasionar patologías severas en humanos como otomicosis, endoftalmitis y neumonías necrotizantes al colonizar vías respiratorias y otras mucosas [8]. La reproducción sexual de este hongo se basa en la producción de ascosporas contenidas en estructuras denominadas ascas. Por otra parte, la reproducción asexual (comúnmente utilizada para la conquista de diferentes hábitats) se da con la producción de conidios en estructuras especializadas llamadas conidióforos [9].

El género Penicillium es ampliamente utilizado en la industria alimenticia en distintos procesos; quesos cultivados como el Roquefort, Brie, Camembert, Stilton y otros, son producidos a partir de diferentes especies de Penicillium como Penicillium roqueforti en donde estos hongos proveen de una amplia gama de sabores y olores a los quesos por la interacción con sus conidios y micelio [10]. 
La producción industrial de compuestos medicinales es otra de las más fuertes aplicaciones de este género de hongos. Albert Fleming en los años 20's descubrió que Penicillium notatum producía una sustancia capaz de inhibir el crecimiento de otros organismos y la llamó penicilina. En la actualidad, este compuesto es un antibiótico perteneciente a la familia de los betalactámicos, capaz de contraatacar distintas infecciones bacterianas y que es producido industrialmente por este hongo para su empleo tanto en la industria médica con en la investigación. P. chrysogenum aparte de producir la penicilina es capaz de producir metabolitos secundarios como alcaloides, en específico roquefortina $\mathrm{C}$, meleagrina y chrisogina [11].

\section{Ganoderma lucidum}

Es un hongo perteneciente al filo Basidiomycota y a la familia Ganodermataceae. Su cuerpo fructífero generalmente crece en los troncos de angiospermas, posee un color rosado-arcilloso poco homogéneo con zonas de crecimiento concéntricas, al madurar adquiere un color blanco y sus basidiosporas presentan formas elipsoides o de pera [12].

G. lucidum ha sido una de las especies con mayor importancia medicinal gracias a sus efectos anticancerígeno, antimicrobiano, antiinflamatorio, antioxidante y antienvejecimiento [12]. Entre los compuestos activos que posee se encuentran polisacáridos, triterpenoides, fibra dietética, aminoácidos, péptidos, proteínas, alcoholes, fenoles, vitaminas y numerosos minerales. Todos ellos en conjunto le dan las propiedades como potencial tratamiento de diferentes enfermedades tales como hipertensión, úlceras gástricas, diabetes y hepatitis, y, el enfoque de esta parte-de la revisión, el cáncer [13].

En algunas investigaciones se ha reportado que los componentes que poseen una mayor actividad fisiológica contra el cáncer son los polisacáridos y los triterpenos [14,15]. En general, los polisacáridos funcionan como antioxidantes y moduladores del sistema inmune, mientras que los triterpenos principalmente inhiben la proliferación de células cancerígenas [14]. Un triterpeno de importancia es el ácido ganodérico, que posee cuatro isoprenos cíclicos y dos lineales y se encuentra en abundancia en las esporas de G. lucidum [15].

De este ácido ganodérico, existen varios subtipos que generan citotoxicidad y pueden causar apoptosis en diferentes líneas celulares cancerígenas, entre ellos el ácido ganodérico T, ácido ganodérico D y ganoderiol F. Además, se ha encontrado que estas sustancias tienen la capacidad para regular el ciclo celular de las células cancerosas y reducir su potencial de realizar metástasis, impidiendo su proliferación hacia otras partes del cuerpo [15].

Esta inhibición a la metástasis se debe principalmente a que los triterpenoides tienen la capacidad de regular y disminuir la actividad de ciertos genes involucrados en su potencial migratorio en un 20\%. Dichos genes corresponden a HRAS, VIL2, S100A4, MCAM, I2PP2A y FN1, muchos de ellos regulan la producción de proteínas de membrana que permiten la adhesión y el movimiento de las células cancerígenas a otros órganos [16].

Se ha encontrado una variedad de polisacáridos en el cuerpo fructífero, el micelio y las esporas de G. lucidum, que se piensa que los extraídos de distintas partes del hongo inducen a diferentes respuestas del sistema inmune [14]. Sus propiedades antitumorales pueden verse afectadas por el grado de ramificación del micelio, que a su vez influye en su solubilidad. En general, mejoran la respuesta del sistema inmune del cuerpo, ya que incrementa la actividad de los macrófagos y los linfocitos T e induce la secreción de compuestos como el TNF (Factor de Necrosis Tumural). Tienen un efecto antioxidante en las células sanas y suprimen el proceso de angiogénesis, mediante el cual se forman nuevos vasos sanguíneos que nutren el tumor [15]. 
Asimismo, estudios han demostrado que estos compuestos pueden mediar la citotoxicidad de diferentes tipos de células tumorales, incluyendo las líneas celulares de cáncer de pulmón, de próstata y de seno. Además, tienen la capacidad de programar la muerte celular mediante una sobreexpresión del factor de crecimiento endotelial [17].

\section{Trametes versicolor}

Es conocido como el hongo de la pudrición blanca, pertenece al filo Basidiomycota y a la familia Polyporaceae. Tiene un cuerpo fructífero en forma de abanico y una estructura en capas con zonas concéntricas de diferentes colores como el gris y el marrón. Comúnmente se encuentran en bosques secos y crecen sobre madera en descomposición [18], [19].

Este hongo presenta compuestos como el polisacárido PSK, un agente estimulador del sistema inmune, con propiedades anticancerígenas y actividad antioxidante. Además, se ha comprobado que los compuestos activos extraídos del micelio de T. versicolor, pueden ser utilizados para tratar individuos que hayan sido expuestos a una intoxicación hepática con $\mathrm{CCl}_{4}$, debido a las propiedades antioxidantes y citoprotectoras de los metabolitos del hongo, los cuales reducen el estrés oxidativo provocado por los radicales libres del $\mathrm{CCl}_{4}$ y contribuyen a conservar el ambiente óxido-reducción del hígado [18].

Este hongo posee compuestos intracelulares como los intrapolisacáridos (IPS), que tienen la habilidad de inhibir el crecimiento de células tumorales, induciendo su apoptosis. Una manera de obtener una mejor producción de estos compuestos es utilizando tirosol. Se ha demostrado que este funciona como una molécula inductora de la síntesis de IPS en T. versicolor y además fortalece su actividad antitumoral. Esta técnica representa una nueva estrategia para producir polisacáridos fúngicos con actividad medicinal [20].

Al comparar la actividad antitumoral de este hongo con otros como Ganoderma resinaceum y Phungia rufa, se ha obtenido que T. versicolor presenta la mayor capacidad para reducir la proliferación de células tumorales. Esta actividad varía de un hongo a otro debido a las diferentes proporciones del contenido de azúcares y proteínas [21].

\section{Schizophyllum commune}

Perteneciente al género Schizophyllum es parte del filo Basidiomycota. Se ha reportado siendo hospedero de muchos organismos y se ha visto su crecer sobre madera en descomposición o en plantas [22]. S. commune ha sido reconocido por su importancia médica; desde la perspectiva farmacológica, es sumamente importante por la producción del polisacárido "schizophyllan" (SPG), al que se le atribuyen propiedades medicinales considerables [23, 24].

Mirfat [23] indica que algunos extractos de S. commune presentan propiedades curativas contra las infecciones por bacterias y hongos, por lo cual se pueden usar como posibles agentes antimicrobianos. Algunos de los microorganismos probados fueron bacterias patógenas comunes, es decir, Bacillus cereus, Bacillus subtilis, Staphylococcus aureus, Pseudomonas aeuroginosa, Proteus vulgaris, Escherichia coli y otras.

Durante las últimas cuatro décadas, extensos estudios sobre el "schizophyllan" han contribuido a la amplia aplicación del polisacárido biológicamente activo en productos farmacéuticos. Las bioactividades que presenta este compuesto incluyen actividades antineoplásicas, antibacterianas y propiedades antiparasitarias. También se han informado otros efectos fisiológicos, como los efectos hepatoprotectores y antiinflamatorios. Entre estas bioactividades, las más prometedoras son sus actividades antitumorales e inmunobiológicas [25]. 
Komatsu et al. [26] fueron los primeros en descubrir que una solución acuosa de SPG tiene una actividad antitumoral mediada por el huésped contra el Sarcoma 180. SPG fue eficaz contra distintos tumores alogénicos y singénicos en ratones, como carcinomas MM46 y MH-134, BC -47 tumor de vejiga, fibrosarcoma AMC-60 y carcinoma mamario A-755. Dicho compuesto en asociación con agentes quimioterapéuticos también inhibió la leucemia L1210, el melanoma B-16 y el fibrosarcoma Meth-A [25].

Mansour et al. [27] demostraron que el SPG, extraído de S. commune, redujo notablemente la incidencia de carcinomas mamarios y hepáticos, disminuyendo la proliferación celular en un modelo con ratones de carcinogénesis inducida por dimetil benz( $\alpha$ )antoceno (DMBA). Los autores concluyeron que por el nivel de seguridad del SPG y su costo relativamente bajo, además de su capacidad para inhibir carcinomas mamarios y para suprimir las lesiones hepáticas asociadas con el tratamiento con tamoxifeno, se puede continuar con investigaciones preclínicas, para la terapia de cáncer de mama con receptores de estrógenos positivos.

Es necesario estudiar y analizar más las capacidades que tiene $S$. commune y su principal compuesto de interés, el "schizophyllan" para comprobar la efectividad de este y aprovechar al máximo su potencial.

\section{Pleurotus mutilus}

Miembro del filum Basidiomycota y de la familia Pleurotaceae. P. mutilus es un hongo verdadero en forma de seta que presenta las mismas características generales que los demás basidiomicetes: pared celular mayoritariamente compuesta de quitina y glucano, hifas dicarióticas estériles y septadas, reproducción sexual mediante basidios con 4 basidiosporas (en promedio) y asexual mediante conidios, y con un tejido que cubre las láminas con las esporas hasta su maduración llamado himenio [28].

P. mutilus es el principal productor de un grupo de antibióticos conocidos como pleuromutilinas (descubiertos por primera vez en 1950), los cuales son los principales precursores de productos más específicos como lo son las tiamulinas y la valnemulina. Esta familia de antibióticos semisintéticos inhibe la síntesis proteica en ciertas bacterias mediante la unión del compuesto al componente bacteriano de la peptidil-transferasa de la subunidad 505 de los ribosomas; además, presentan baja citotoxicidad en mamíferos, poco impacto a especies no deseadas y un bajo impacto ambiental [29].

La tiamulina es un antibiótico bacteriostático a concentraciones terapéuticas, mostrando actividad a nivel ribosomal $70 S$ donde se une principalmente a la subunidad 505 . Este antibiótico inhibe la producción de proteínas bacterianas mediante la producción de complejos de iniciación con inactivación bioquímica en donde no se permite la elongación del péptido. Por otra parte, este antibiótico ha mostrado actividad en el laboratorio contra bacterias como: Brachyspira hyodysenterieae, Brachyspira pilosicoli, Lawsonia intracellularis y Mycoplasma spp [30].

Como es común, además, se ha visto resistencia en especies de Brachyspira la cual se cree que es producto de mutaciones en los sitios de unión ribosomal o a una desensibilización a algunas otras pleuromutilinas. En general, la tiamulina presenta beneficios o usos en veterinaria, ya que se ha comprobado que tiene efectos en porcinos y conejos para el tratamiento y prevención de padecimientos como disentería porcina, ileitis, neumonía enzoótica y EEC (Enterocolitis Epizoótica del Conejo) [30].

Por otra parte, la biomasa producida en fermentación sólida de P. mutilus considerada como desecho luego de la producción y extracción de los antibióticos de interés, muestra propiedades de biorremediación al ser utilizada como bio-adsorbente de metales pesados y radioactivos 
como lo es el uranio (presente en fuentes de contaminación acuífera), cobre y algunos iones ferrosos de cianuro. Esta aplicación es de gran interés a nivel global, ya que se tratan dos aristas ambientales de alto impacto como lo son: el uso y aprovechamiento de desechos industriales y la eliminación de compuestos o elementos contaminantes en el medio ambiente [31].

\section{Lentinula edodes}

Es conocido como Shiitake, del filo Basidiomicota y de la familia Tricholomataceae. Su cuerpo fructífero tiene forma de seta de color café claro a oscuro y crece sobre madera en descomposición. Es originario de Asia, y producido en América Central y el Caribe [32]. Este hongo es reconocido por su valor nutricional, debido a su alto contenido de fibras y proteínas que contribuyen a la salud del ser humano, ya que previene enfermedades gastrointestinales, reduce el nivel de colesterol, mejora la presión sanguínea y participa en la prevención del cáncer. Además de esto, se han aislado otros metabolitos del Shiitake que presentan actividad antiinflamatoria, antitumoral, antiviral, y hepatoprotectora, convirtiendo a $L$. edodes en uno de los mejores alimentos funcionales [33].

Uno de los metabolitos más importantes del Shiitake es la eritadenina, la cual disminuye la concentración del colesterol en el cuerpo al modificar el procesamiento hepático de los lípidos. Estos efectos se han probado en ratones al proporcionarles una dieta suplementada con eritadenina, lo cual supone que los componentes de este hongo pueden ser utilizados para tratar hipercolesterolemia en seres humanos [33] [34].

El lentinan es un polisacárido aislado de L.edodes que se caracteriza por su actividad antitumoral debido a que posiblemente activa una respuesta inmune en el huésped, por lo cual ya se utiliza en el tratamiento de diferentes enfermedades cancerígenas; además, se ha investigado otras actividades biológicas del lentinan como la activación de respuestas inflamatorias no específicas [35].

Por otra parte, el contenido de polisacáridos, glucanos y fenoles de este hongo han sido estudiados encontrando propiedades antioxidantes debido a la estructura de anillos aromáticos de estos compuestos, protegiendo a las células de ser dañadas por los radicales libres que se producen por agentes externos [36]. Otros compuestos bioactivos extraídos del Shiitake son el alfa-tocoferol (vitamina E), el ácido oléico, el ergosterol y el ácido butírico; compuestos que en general pueden actuar como antioxidantes y poseen actividad anti-arteriosclerótica debido a su participación en el metabolismo lipídico [37].

\section{Psilocybe cubensis}

Los hongos psicoactivos han sido utilizados por civilizaciones antiguas durante miles de años con fines religiosos, culturales y medicinales [38]. Uno de los más comunes en el continente americano es $P$. cubensis del filo Basidiomicota y de la familia Strophariaceae. Es pequeño, posee un tallo largo, delgado y fibroso, su sombrerillo es convexo y puede tener coloración azul oscuro conforme alcanza la madurez [39]. Si bien no es utilizado para tratar padecimientos físicos, se ha reportado su aplicación en la psicología clínica para tratar condiciones que afectan la salud mental gracias a sus efectos psicotrópicos.

El principal metabolito secundario que tiene actividad psicotrópica es la psilocibina (0-fosforil-4hidroxi-N-dimetiltriptamina), un alcaloide que al ser ingerido es sometido a una desfosforilación y se transforma en psilocina (4-hidroxi-N dimetiltriptamina) [38]. Éste último posee un potencial alucinógeno mayor, ya que atraviesa las barreras entre los capilares y las neuronas del cerebro con mayor facilidad. Induce un estado de percepción alterado, sensación de euforia, alteración 
de los colores, alucinaciones y despersonalización. Su intensidad varía de acuerdo con la especie que se utiliza, la cantidad ingerida, la sensibilidad al compuesto y demás factores [40]. Algunos de los efectos físicos que se pueden presentar son el aumento de las palpitaciones del corazón, hipertensión y temperatura corporal, sin embargo, no generan toxicidad. El mayor riesgo de ingerir psilocibina es la incapacidad para lidiar con la pérdida temporal de estabilidad psicológica [39].

La estructura molecular de la psilocina es muy similar a la de la serotonina (5-HT), y se ha encontrado que los efectos psicóticos resultan de su interacción con receptores $5-\mathrm{HT}_{1 \mathrm{~A}}, 5-\mathrm{HT}_{2 \mathrm{~A}}$ y $5-\mathrm{HT}_{2 \mathrm{C}}$ [41]. Su uso puede tener una influencia positiva durante sesiones de psicoterapia, lo cual ayuda a mejorar el humor y disminuir diferentes síntomas de enfermedades mentales, como el trastorno obsesivo compulsivo (TOC), depresión, ansiedad y adicciones [42].

Existen reportes de pacientes con TOC que después de ingerir $P$. cubensis presentan un alivio de sus síntomas durante varios meses, incluso si su experiencia estando bajo los efectos psicoactivos les generó ansiedad, y luego de un consumo frecuente lograron eliminar por completo sus pensamientos intrusivos[42]. Del mismo modo, su uso en el tratamiento de otros padecimientos psiquiátricos relacionados con desórdenes en los niveles de serotonina en el cerebro ha sido estudiado en algunos casos. Se ha tenido éxito eliminando síntomas ansiosos y depresivos en pacientes con enfermedades terminales, así como reduciendo el consumo de sustancias dañinas como el alcohol y drogas $[43,44]$.

\section{Conclusiones}

Los hongos ofrecen una gran variedad de metabolitos que pueden explotarse para aplicarlos a la medicina, siendo una de las mejores alternativas naturales para combatir enfermedades tanto comunes como aquellas que aún no tienen un tratamiento específico. En estos organismos se pueden encontrar compuestos como polisacáridos, triterpenos, antibióticos, proteínas y otros, cuyas funciones más importantes son la actividad anticancerígena o antitumoral, antiinflamatoria, antioxidante, antibiótica, algunos tienen un efecto hepatoprotector y otros pueden utilizarse como psicotrópicos para tratar enfermedades mentales.

A pesar de que el área centroamericana posea gran diversidad de especies fúngicas, su aplicación en la actividad medicinal no ha sido explotada como en otras partes del mundo; lo cual demuestra que aún existen muchas posibilidades de explotación científica para el beneficio de la humanidad.

\section{Agradecimientos}

Se agradece al M.Sc. William Rivera Méndez, profesor e investigador de la Escuela de Biología y del Centro de Investigación en Biotecnología del Instituto Tecnológico de Costa Rica por su aporte, acotaciones, recomendaciones y apoyo con esta publicación. 


\section{Referencias}

[1] M. Vásquez, "La relación del ser humano con su entorno. Dimensión Ética y Social", Stuidiositas, vol. 2, no. 1, pp. 55-60, 2017.

[2] UACJ, "Introducción a los hongos" Hoja Técnica de Divulgación Científica, vol. 2, no. 1, pp.1-4, 2012.

[3] E. Velasquez, "Asociación Micológica" Fungipedia, 15 Mayo 2015. [En línea]. Disponible en: https://www.fungipedia.org/103-mi-blog/general/379-la-medicina-y-la-micologia-historia.html. [Último acceso: 2018 Mayo 10]

[4] N. Bernardo y J. Hermoso, "La casualidad que llevó al descubrimiento de la penicilina" CISC, Abr. 2015.

[5] T. Urribarren, E. Bazán y L. Rosio, "Generalidad de la Micología" Dpto. de Microbiología y ParasitologíaRecursos de Micología, vol. 3, no. 2, pp.3-9, 2017.

[6] A. Ruiz, <Los hongos y su importancia>2014.[En línea]. Disponible en: http://www.museocostarica.go.cr/ es_cr/ent-rese-./los-hongos-y-su-importancia-3.html?|temid=62

[7] D. Ellis, "Penicillium" The University of Adelaide, 15 Diciembre 2016. [En línea]. Disponible en: https://mycology.adelaide.edu.au/descrptions/hyphomycetes/penicillium/. [Último acceso: 30 Abril 2018].

[8] T.K. Plewaj y E. Lonc, "Molecular identification and biodiversity of potencial allergenic molds (Aspergillus and Penicillium) in the poultry house: first report" Aerobiología, vol.30, no. 4, pp.445-451, 2014.

[9] F. Francis, K. Jaber, F. Colinet, D. Potetelle y E. Haubruge, "Purification of newfungal mannose-specific lectin from Penicillium chrysogenum and its aphidicial properties" Fungi Biol, vol. 115, no. 1, pp- 1093-1099, 2012.

[10] V. Greenwood, "El fascinante mundo del interior del queso" BBC Mundo, 4 Diciembre 2014.

[11] EcuRed, "Penicillium," 2017. [En línea]. Disponible en: https://www.ecured.cu/Penicillium

[12] L.-W. Zhou, Y. Cao, S.-H. Wu, J. Vlasák, D.-W. Li, M.-J. Li, and Y.-C. Dai, "Global diversity of the Ganoderma lucidum complex (Ganodermataceae, Polyporales) inferred from morphology and multilocus phylogeny," Phytochemistry, vol. 114, pp. 7-15, Oct. 2014.

[13] P. Batra, A. K. Sharma, and R. Khajuria, "Probing Lingzhi or Reishi Medicinal Mushroom Ganoderma lucidum (Higher Basidiomycetes): A Bitter Mushroom with Amazing Health benefits," Int. J. of Medicinal Mushrooms, vol.15, no. 2, pp. 127-142, 2013.

[14] G.-S. Wu, J.-J. Guo, J.-L. Bao, X.-W. Li, X.-P. Chen, J.-J. Lu, and Y.-T. Wang, "Anti-cancer properties of triterpenoids isolated from Ganoderma lucidum - a review," Expert Opin. Investig. Drugs, vol. 22, no. 8, pp. 1-12, Aug. 2013

[15] C.-H. J. Kao, A. C. Jesuthasan, K. S. Bishop, M. P. Glucina, and L. R. Ferguson, "Anti-cancer activities of Ganoderma lucidum: active ingredients and pathways," Functional Foods in health and Disease, vol. 3, no. 2, pp. 48-65, Feb. 2013.

[16] J. Loganathan, J. Jilang, A. Smith, A. Jedinak, A. Thyagarajan-Sahu, G. E. Sandusky, H. Nakshatri, and D. Sliva, "The mushroom Ganoderma lucidum suppresses breast-to-lung cancer metastasis through the inhibition of pro.invasive genes," Int. J. of Oncology, vol. 44, no. 6, pp. 2009-2015, Apr. 2014.

[17] L. Zengenni, G. Yu-Tong, Y. You-Jin, W. Ren-Cai, H. Qiu-Long, Y. X. Xing, "Ganoderma lucidum polysaccharides target a Fas/Caspase dependant pathway to induce apoptosis in human colon cancer cells," Asian Pacific J. of Cancer Prevention, vol. 15, no. 9, pp. 3981-3986, 2014

[18] P. Rojas-Franco, E. Cano-Europa, R. Ortiz-Butron, R. Valenzuela-Garza, V. Blas-Valdivia, y M. Franco-Colin, "Efecto hepatoprotector del micelio de Trametes versicolor en un modelo de ratón de la cepa NIH," Rev. Mex. Cienc Farm, vol. 46, no. 1, pp. 56-63, 2015.

[19] N. Ubau, "Distribución de Hongos Medicinales en las Macro Regiones Pacífico y Centro de Nicaragua 2006 2011," Rev. Univ. Cienc., vol. 9, no. 15, pp. 4-16, 2016.

[20] K. F. Wang, K. Sui, C. Guo, y C.Z. Liu, "Improved production and antitumor activity of intracellular proteinpolysaccharide from Trametes versicolor by the quorum sensing molecule-tyrosol," J. Funct. Foods, vol. 37, pp. 90-96, 2017.

[21] A. Silva, A. Miranda, E. Fernandes, S. Santos, I. Fraga, D. Santos y R. Bezerra, "Endopolysaccharides from Ganoderma resinaceum, Phlebia rufa, and Trametes versicolor Affect Differently the Proliferation Rate of HepG2 Cells," Appl Biochem Biotechnol, vol. 169, pp. 1919-1926, 2013.

[22] S. Vázquez-Mendoza. "Nuevo hospedero del hongo Schizophyllum commune en América". Rev. Mexicana de Biodiversidad. vol. 84, no. 2, pp.661-663, 2013.

[23] A. Mirfat, A, Noorlidah, S, Vikineswary, . "Antimicrobial activities of split gill mushroom Schizophyllum commune". American Journal of Research Communication, vol. 2, no. 7, pp. 113-124, 2014.

[24] L. Castro, M. Álvarez \& E. Martínez. "Case report of Schizophyllum commune sinusitis in an immunocompetent patient”. Rev Colombia Médica. vol. 41, no. 1, pp.71-76, 2010. 
[25] Y. Zhang, H. Kong, Y. Fang, K. Nishinari, \& G. Phillips. "Schizophyllan: A review on its structure, properties, bioactivities and recent developments". Bioactive Carbohydrates and Dietary Fibre. vol.1, no.1, pp. 53-71, 2013.

[26] N. Komatsu, S. Okubo, S. Kikumoto, K. Kimura, G. Saito, \& S. Sakai. "Host-mediated antitumor action of schizophyllan, a glucan produced by Schizophyllum commune." Gann, pp. 137-144, 1969.

[27] A. Mansour, A. Daba, N. Baddour, M. El-Saadani, E. Aleem. "Schizophyllan inhibits the development of mammary and hepatic carcinomas induced by 7,12 dimethylbenzo(a) antharacene and decreases cell proliferation: comparison with taxoxifen." Rev. Cancer Research and Clinical Oncology. vol. 138, no. 9, pp.1579-1596, 2012.

[28] IMI, <International Mycotherapy Institute>, 15 Diciembre 2014. [En línea]. Disponible: http://www.imispain. com/los-beneficios-del-genero-pleurotus/. [Último acceso: 2018 Mayo 10].

[29] S. Sun, L. Ai, H. Zhang, C. Weng, C. Lai y L. Liu, «Enhanced production of pleuromutilin by Pleurotus mutilus and study on its molecular structure» Food Chemistry, vol. 230, pp. 350-353, 2017.

[30] A. Pintaluba, «Composición Cualitativa y Cuantitativa,» Aditivos y Medicamentos para la sanidad y nutrición animal, pp. 2-5, 2014.

[31] M. Mezaguer, N. El Hayet Kamel, H. Lounici y Z. Kamel, «Characterization and properties of Pleurotus mutilus fungal biomass as adsorbent of the removal of Uranium ( $\mathrm{VI}$ ) from uranium leachate» Journal Radioanalysis Nuclear Chemistry, vol. 295, pp. 393-403, 2013.

[32] M.A. Barradas, "Efecto de diferentes sustratos y cepas sobre las características físico-químicas y compuestos con actividad antioxidante en carpóforos de shiitake (Lentinula edodes)," Tesis para obtener el grado de Maestro en Ciencias Alimentarias, Universidad Veracruzana, Xalapa, Veracruz, México, Octubre, 2015.

[33] O.M. Rivera, W. Albarracín y M. Lares, "Componentes Bioactivos del Shiitake (Lentinula edodes Berk. Pegler) y su impacto en la salud," AVFT, vol. 36, no. 3, pp. 67-71, 2017.

[34] H. Yang, I. Hwang, S. Kim, E.J. Hong y E.B. Jeung, "Lentinus edodes promotes fat removal in hypercholesterolemic mice," Exp Ther Med, vol. 6, no. 6, pp. 1409-1413, Dic, 2013.

[35] T.C. Finimundy, G. Gambato, R. Fontana, M. Camassola, M. Salvador, S. Moura, J. Hess, J.A.P. Henriques, A.J.P. Dillon y M. Roesch-Ely, "Aqueous extracts of Lentinula edodes and Pleurotus sajor-caju exhibit high antioxidant capability and promising in vitro antitumor activity," Nutr Res, vol. 33, pp. 76-84, 2013.

[36] M. Kozarski, A. Klaus, M. Niksic, M.M. Vrvic, N. Todorovic, D. Jakovljevic, y L.J.L.D. Van Griensven, "Antioxidative activities and chemical characterization of polysaccharide extracts from the widely used mushrooms Ganoderma applanatum, Ganoderma lucidum, Lentinus edodes and Trametes versicolor," J Food Compost Anal, vol. 26, pp. 144-153, 2012

[37] M.A. Rahman, N. Abdullah, N. Aminudin, "Lentinula edodes (shiitake mushroom): An assessment of in vitro anti-atherosclerotic bio-functionality," Saudi J Biol Sci, 2016.

[38] F. J. Carod-Artal, "Alucinógenos en las culturas precolombinas mesoamericanas," Neurología, vol. 30, no. 1, pp. 42-49, Feb. 2015.

[39] G. P. Menser, Hallucinogenic and Poisonous Mushroom Field Guide. California, USA: Ronin Publishing.

[40] G. Burillo-Putze, E. López-Briz, B. Climent-Díaz, P. Munné-Mas, S. Nogue-Xarau, M.A. Pinillos, R.S. Hoffman, "Drogas emergentes (III): plantas y hongos alucinógenos," Anales. Sis. San. Navarra, vol. 36, no. 3, Dic. 2013.

[41] K. Stebelska, "Fungal Hallucinogens Psilocin, Ibotenic Acid, and Muscimol: Analytical Methods and Biological Activities," Ther. Drug Monit., vol. 35, no. 4, pp. 420-442, Aug. 2013.

[42] J. A. Wilcox, "Psilocybin and Obsessive-Compulsive Disorder," J. of Psychoactive Drugs, vol. 46, no. 5, pp. 393-395, Nov. 2014.

[43] A. K. Loizaga-Velder y A. Loizaga-Pazzi, "El potencial terapéutico de los alucinógenos en el tratamiento de las adicciones," Rev. Electrónica de Psicología Iztacala, vol. 20, no. 3, pp. 924-953, Sep. 2017.

[44] F. Tylš, T. Páleníček y J. Horáček, "Psilocybin-summary of knowledge and new perspectives," European Neuropsychopharmacology, vol. 24, no. 3, pp. 342-356, Mar. 2014. 